Article

\title{
Stability of Sets Criteria for Impulsive Cohen-Grossberg Delayed Neural Networks with Reaction-Diffusion Terms
}

\author{
Gani Stamov ${ }^{1,+, \ddagger}$, Stefania Tomasiello ${ }^{2, \ddagger} \mathbb{0}$, Ivanka Stamova ${ }^{3, *, \ddagger}$ and Cvetelina Spirova ${ }^{1, \ddagger}$ \\ 1 Department of Mathematics, Technical University of Sofia, 8800 Sliven, Bulgaria; gstamov@abv.bg (G.S.); \\ cvetelina2802@abv.bg (C.S.) \\ 2 Institute of Computer Science, University of Tartu, Narva mnt 18, 51008 Tartu, Estonia; \\ stefania.tomasiello@ut.ee \\ 3 Department of Mathematics, University of Texas at San Antonio, San Antonio, TX 78249, USA \\ * Correspondence: ivanka.stamova@utsa.edu \\ + Current address: Department of Mathematics, University of Texas at San Antonio, \\ San Antonio, TX 78249, USA. \\ $\ddagger \quad$ These authors contributed equally to this work.
}

Received: 3 December 2019; Accepted: 18 December 2019; Published: 21 December 2019 updates

\begin{abstract}
The paper proposes an extension of stability analysis methods for a class of impulsive reaction-diffusion Cohen-Grossberg delayed neural networks by addressing a challenge namely stability of sets. Such extended concept is of considerable interest to numerous systems capable of approaching not only one equilibrium state. Results on uniform global asymptotic stability and uniform global exponential stability with respect to sets for the model under consideration are established. The main tools are expansions of the Lyapunov method and the comparison principle. In addition, the obtained results for the uncertain case contributed to the development of the stability theory of uncertain reaction-diffusion Cohen-Grossberg delayed neural networks and their applications. Moreover, examples are given to demonstrate the feasibility of our results.
\end{abstract}

Keywords: stability of sets; Cohen-Grossberg neural networks; impulsive perturbations; delays

\section{Introduction}

The well known Cohen-Grosberg type neural networks (CGNNs) introduced in 1983 [1] have been widely investigated due mainly to their numerous applications in science and engineering [2-4]. It is also well known that some powerful from the applied point of view neural network models, such as cellular neural networks, bidirectional neural networks, Hopfield neural networks, can be considered as special cases of CGNNs. Later on, the investigations on delayed CGNNs with constants and time-varying delays also had increased rapidly [5-7] including some recent results [8-10]. In addition, the subject of reaction-diffusion delayed CGNNs has been studied in [11-14]. Indeed, considering the effect of reaction-diffusion terms on the neural network dynamics is essential [15-19]. As it is mentioned in [11] "... the whole structure and dynamic behavior of neural networks not merely dependent on the evolution time of each variables but also intensively dependent on its position (space)."

On the other hand, considering impulsive perturbations in CGNNs is a very hot topic of interest [20-24]. It has been found that, because of some instantaneous perturbations, the behaviors of many real-word systems are not continuous processes, and such processes can be modelled by impulsive differential equations [25-29]. Therefore, it is crucial to study the dynamic properties of impulsive reaction-diffusion CGNNs, and numerous excellent qualitative results have been published. 
See, for example, [30-35] and the references therein. In addition, the impulsive control is found to be very efficient control approach in numerous applied problems [36-44].

Asymptotic stability of equilibrium states is one of the most important qualitative properties that has to be guaranteed in most of the applications of neural network models designed. This is why most of the existing results on neural network systems are related to asymptotic or exponential stability of equilibrium states [2-4,8-10,12,16,17,22-24,30,33,35,37,45].

However, even in the pioneering work of Cohen and Grossberg [1], systems capable of approaching not only one, but infinitely many equilibrium points in response to arbitrary initial data, have been considered. For such systems, researchers proposed the concept of stable (asymptotically stable) sets (or manifolds) [46]. This extended stability concept is also of a significant importance in numerous applications, when the authors investigate the global asymptotic behavior of invariant sets, invariant manifolds, or of sets of a general nature [47-53]. We note that the notion of stability of sets includes as a special case stability of an equilibrium, stability of zero solution, stability of moving manifolds, etc. Thus, stability of sets is one of the most important notions in the stability theory. However, besides the great possibilities for applications, the topic of stable sets has not been studied for impulsive reaction-diffusion CGNNS and this is the main objective of the paper.

On the other hand, the effect of some uncertain parameters on the qualitative behavior of CGNNs has been studied by several authors. See $[5,7,21]$ and the references therein. In this paper, we will further extend the existing robust stability results to the stability of sets case. Indeed, uncertainty associated with concrete systems parameters arises from modeling assumptions, the lack of knowledge or information of the real-world situations, noise factors, etc. Hence, finding efficient conditions that will guarantee the stability behavior of a uncertain system is a question of a great importance. Therefore, the dynamics of uncertain systems has long been and will continue to be one of the dominant themes in mathematics and mathematics applications [54-58].

The paper is organized as follows. In Section 2, the impulsive reaction-diffusion CGNN model in addition to the basic notations and preliminaries are introduced. The notion of stability of sets with respect to the model under consideration is defined. Section 3 offers our main stability of sets results. In Section 4, the effect of some uncertain parameters is considered, and a robust stability analysis is also conducted. In Section 5, to show the effectiveness of the obtained results, two examples are given. Finally, some conclusion remarks are drawn in Section 6.

\section{Preliminaries}

Let $\mathbb{R}^{n}$ be the $n$-dimensional Euclidean space with norm $\|x\|=\left(\sum_{k=1}^{n} x_{k}^{2}\right)^{1 / 2}$ of $x=$ $\left(x_{1}, x_{2}, \ldots, x_{n}\right)^{T} \in \mathbb{R}^{n}, \Omega$ be an open and bounded set in $\mathbb{R}^{n}$ that contains the origin has a smooth boundary $\partial \Omega$ and the measure expressed by mes $\Omega>0$, and let $\mathbb{R}_{+}=[0, \infty)$. For $u(t, x)=$ $\left(u_{1}(t, x), u_{2}(t, x), \ldots, u_{m}(t, x)\right)^{T} \in \mathbb{R}^{m}$, we also consider the following norm:

$$
\|u(t, x)\|_{2}=\left[\int_{\Omega} \sum_{i=1}^{m} u_{i}^{2}(t, x) d x\right]^{1 / 2} .
$$

We note that the space $L^{2}(\Omega)$ of all real functions on $\Omega$, which are $L^{2}$ for the Lebesgue measure, is a Banach space with respect to the above norm [13,16,31,32,34].

In this research, we will investigate some qualitative characteristics of the processes determined by the following delayed reaction-diffusion CGNN that is subject to short-term impulsive perturbations at fixed moments of time

$$
\frac{\partial u(t, x)}{\partial t}-\nabla(D(t, x) \circ \nabla u(t, x))-F(t, u(t, x), u(t-\tau, x))=\eta(t, x),
$$


where $t \in \mathbb{R}, x \in \Omega, u=u(t, x)$ and:

(i) $D(t, x)=\left(D_{i q}(t, x)\right)_{m \times n}$ is an $m \times n$ matrix with entries the functions $D_{i q}(t, x), i=$ $1,2, \ldots, m, q=1,2, \ldots, n, \nabla$ is the gradient operator, $\nabla=\left(\frac{\partial}{\partial x_{1}}, \frac{\partial}{\partial x_{2}}, \ldots, \frac{\partial}{\partial x_{n}}\right), \nabla u_{i}=$ $\left(\frac{\partial u_{i}}{\partial x_{1}}, \frac{\partial u_{i}}{\partial x_{2}}, \ldots, \frac{\partial u_{i}}{\partial x_{n}}\right), i=1,2, \ldots, m$ and $\nabla u=\left(\nabla u_{1}, \nabla u_{2}, \ldots, \nabla u_{m}\right)^{T}$, "." is the inner product, "०" denotes the Hadmard product [58] of the matrices $D$ and $\nabla u$;

(ii) $F(t, u(t, x), u(t-\tau, x))=-A(u(t, x))[B(u(t, x))-I(t, x)-C(t) f(u(t, x))-W(t) g(u(t-$ $\tau, x))], A(u(t, x))=\operatorname{diag}\left(a_{1}(t, x), a_{2}(t, x), \ldots, a_{m}(t, x)\right)$ is a diagonal matrix with entries $a_{i} \in$ $C\left[\mathbb{R} \times \mathbb{R}^{n}, \mathbb{R}_{+}\right], \quad i=1,2, \ldots, m, B(u(t, x))=\operatorname{diag}\left(b_{1}(t, x), b_{2}(t, x), \ldots, b_{m}(t, x)\right), \quad b_{i} \in C[\mathbb{R} \times$ $\left.\mathbb{R}^{n}, \mathbb{R}\right], i=1,2, \ldots, m, I(t, x)=\left(I_{1}(t, x), I_{2}(t, x), \ldots, I_{m}(t, x)\right)^{T}, I_{i} \in C\left[\mathbb{R} \times \mathbb{R}^{n}, \mathbb{R}\right], i=1,2, \ldots, m$, $C(t)=\left(c_{i j}(t)\right)_{m \times m}, \quad c_{i j} \in C[\mathbb{R}, \mathbb{R}], W(t)=\left(w_{i j}(t)\right)_{m \times m}, \quad w_{i j} \in C[\mathbb{R}, \mathbb{R}], f(u(t, x))=$ $\left(f_{1}\left(u_{1}(t, x)\right), f_{2}\left(u_{2}(t, x)\right), \ldots, f_{m}\left(u_{m}(t, x)\right)\right)^{T}, f_{j} \in[\mathbb{R}, \mathbb{R}], j=1, \ldots, m, g(u(t-\tau, x))=\left(g_{1}\left(u_{1}(t-\right.\right.$ $\left.\left.\left.\tau_{1}(t), x\right)\right), g_{2}\left(u_{2}\left(t-\tau_{2}(t), x\right)\right), \ldots, g_{m}\left(u_{m}\left(t-\tau_{m}(t), x\right)\right)\right)^{T}, g_{i} \in C[\mathbb{R}, \mathbb{R}], \quad i=1, \ldots, m, \tau_{j} \in$ $C\left[\mathbb{R}, \mathbb{R}_{+}\right], t>\tau_{j}, j=1, \ldots, m, 0 \leq \tau_{j}(t) \leq \tau, \frac{d \tau_{j}(t)}{d t}<\delta_{j}\left(\tau>0, \delta_{j}<1\right) ;$

(iii) $\eta(t, x)=\sum_{k=1,2, \ldots,} J_{k}(u(t, x)) \delta\left(t-t_{k}\right), J_{k}=\operatorname{diag}\left(J_{1 k}, J_{2 k}, \ldots, J_{m k}\right), J_{i k} \in \mathbb{R}, i=1, \ldots, m, k=$ $1,2, \ldots, \delta(t)$ is the impulsive Dirac-type function with impulsive points $t_{k}, k=1,2, \ldots, 0<t_{1}<t_{2}<$ $\cdots<t_{k}<t_{k+1}<\ldots, \lim _{k \rightarrow \infty} t_{k}=\infty$.

From the presentation of the term $\eta(t, x)$, it follows that, for $x \in \Omega$ and $t \neq t_{k}, k=1,2, \ldots$, we have $\eta(t, x)=0$. Hence, we obtain (see [34,42])

$$
u\left(t_{k}^{+}, x\right)-u\left(t_{k}, x\right)=J_{k}\left(u\left(t_{k}, x\right)\right),
$$

where $u\left(t_{k}^{+}, x\right)=\lim _{h \rightarrow 0^{+}} u\left(t_{k}+h, x\right), x \in \Omega$.

Using the above notations, the matrix impulsive delayed reaction-diffusion CGNN model (1) can be represented as

$$
\left\{\begin{aligned}
& \frac{\partial u_{i}(t, x)}{\partial t}= \sum_{q=1}^{n} \frac{\partial}{\partial x_{q}}\left(D_{i q} \frac{\partial u_{i}(t, x)}{\partial x_{q}}\right)-a_{i}\left(u_{i}(t, x)\right)\left[b_{i}\left(u_{i}(t, x)\right)\right. \\
&-I_{i}(t, x)-\sum_{j=1}^{m} c_{i j}(t) f_{j}\left(u_{j}(t, x)\right) \\
&\left.-\sum_{j=1}^{m} w_{i j}(t) g_{j}\left(u_{j}\left(t-\tau_{j}(t), x\right)\right)\right] t \neq t_{k}, \\
& u_{i}\left(t_{k}^{+}, x\right)-u_{i}\left(t_{k}, x\right)=J_{i k}\left(u_{i}\left(t_{k}, x\right)\right),
\end{aligned}\right.
$$

where $i=1,2, \ldots, m, m \geq 2, k=1,2, \ldots, t>0, x=\left(x_{1}, x_{2}, \ldots, x_{n}\right)^{T} \in \Omega, u_{i}(t, x)$ denotes the state of the $i$-th neural unit, $\tau_{j}(t)$ is the transmission time-varying delay of the $i$-th unit, $a_{i}\left(u_{i}(t, x)\right)$ is an amplification function, $b_{i}\left(u_{i}(t, x)\right)$ is an appropriately behaved function, $c_{i j}(t)$ and $w_{i j}(t)$ are the connection weight matrices, $I_{i}(t, x)$ is the external input of the $i$-th neural unit, $f_{j}\left(u_{j}(t, x)\right)$ and $g_{j}\left(u_{j}(t-\right.$ $\left.\left.\tau_{j}(t), x\right)\right)$ are the activation functions of the $j$-th neuron, the smooth functions $D_{i q}=D_{i q}(t, x) \geq 0$ are the transmission diffusion coefficients along the $i$-th neuron. The points $\left\{t_{k}\right\}, k=1,2, \ldots$ are the impulsive moments at which abrupt changes of the state $u_{i}(t, x)$ from positions $u_{i}\left(t_{k}, x\right)$ into the positions $u_{i}\left(t_{k}^{+}, x\right)$ are observed and $J_{i k}\left(u_{i}(t, x)\right)$ are the impulsive functions that measure the impulsive control effects on the node $u_{i}(t, x)$ at the instants $t_{k}$ if we consider the function $\eta(t, x)$ as a control impulsive function.

Let $J \subset \mathbb{R}_{+}$be an interval. Furthermore, we will use classes of functions of the following types: 
$P C\left[J \times \Omega, \mathbb{R}^{m}\right]=\left\{\bar{\sigma}: J \times \Omega \rightarrow \mathbb{R}^{m}: \bar{\sigma}(t, x)\right.$ is continuous everywhere on the domain except at points of the type $\left(t_{k}, x\right) \in J \times \Omega$ wherever $\bar{\sigma}\left(t_{k}^{-}, x\right)$ and $\bar{\sigma}\left(t_{k}^{+}, x\right)$ exist and $\left.\bar{\sigma}\left(t_{k}^{-}, x\right)=\bar{\sigma}\left(t_{k}, x\right)\right\}$;

$\mathcal{P C}$ is the set of all piecewise continuous functions $\varphi=\left(\varphi_{1}, \varphi_{2}, \ldots, \varphi_{m}\right)^{T}$ from $[-\tau, 0] \times \Omega$ to $\mathbb{R}^{m}$, such that $\varphi_{i}\left(\xi^{+}, x\right)$ and $\varphi_{i}\left(\xi^{-}, x\right)$ exist and $\varphi_{i}\left(\xi^{-}, x\right)=\varphi_{i}(\xi, x), i=1,2 \ldots, m$, for all points $\xi \in[-\tau, 0]$ which must be a finite number;

$\mathcal{P C B}$ is the set of all functions $\varphi \in \mathcal{P C}$ that are bounded.

Let $\varphi_{0}=\left(\varphi_{01}, \varphi_{02}, \ldots, \varphi_{0 m}\right)^{T} \in \mathcal{P C B}$. We will consider the following boundary and initial conditions associated with (2):

$$
\begin{gathered}
u_{i}(t, x)=0, t \in[-\tau, \infty), x \in \partial \Omega, i=1,2, \ldots, m, \\
u_{i}(\xi, x)=\varphi_{0 i}(\xi, x), \xi \in[-\tau, 0], x \in \Omega, i=1,2, \ldots, m .
\end{gathered}
$$

We denote by $u(t, x)=u\left(t, x ; \varphi_{0}\right)$ the solution of the Initial Boundary Problem (IBP) (2), (3), (4). Note that, according to the theory of impulsive neural networks [20-22,29-44], the solutions

$$
u(t, x)=\left(u_{1}(t, x), u_{2}(t, x), \ldots, u_{m}(t, x)\right)^{T}
$$

of IBPs (2), (3), (4) are piecewise continuous functions that have jump discontinuities at the moments $t_{k}, k=1,2, \ldots$, and

$$
u_{i}\left(t_{k}^{-}, x\right)=u_{i}\left(t_{k}, x\right), u_{i}\left(t_{k}^{+}, x\right)=u_{i}\left(t_{k}, x\right)+J_{i k}\left(u_{i}\left(t_{k}, x\right)\right), x \in \Omega, k=1,2, \ldots
$$

For more details about the theory of reaction-diffusion CGNN systems with impulsive perturbations, we refer to [30-35].

Throughout this paper, we assume that the following conditions are satisfied:

Hypothesis 1 (H1). The functions $a_{i}, i=1,2, \ldots, m$, are positive, continuous and bounded, i.e., there exist constants $\underline{a}_{i}$ and $\bar{a}_{i}$ such that $1<\underline{a}_{i} \leq a_{i}(\chi) \leq \bar{a}_{i}$ for $\chi \in \mathbb{R}$.

Hypothesis 2 (H2). The functions $b_{i}, i=1,2, \ldots, m$, are continuous and there exist positive constants $B_{i}$ with

$$
\frac{b_{i}\left(\chi_{1}\right)-b_{i}\left(\chi_{2}\right)}{\chi_{1}-\chi_{2}} \geq B_{i}>0
$$

for $\chi_{1}, \chi_{2} \in \mathbb{R}, \chi_{1} \neq \chi_{2}$.

Hypothesis 3 (H3). The activation functions $f_{j}$ and $g_{j}$ are continuous and Lipschitz, i.e., there exist positive constants $L_{j}, M_{j}, j=1,2, \ldots, m$, with

$$
\begin{aligned}
& \left|f_{j}\left(\chi_{1}\right)-f_{j}\left(\chi_{2}\right)\right| \leq L_{j}\left|\chi_{1}-\chi_{2}\right|, \\
& \left|g_{j}\left(\chi_{1}\right)-g_{j}\left(\chi_{2}\right)\right| \leq M_{j}\left|\chi_{1}-\chi_{2}\right|
\end{aligned}
$$

for all $\chi_{1}, \chi_{2} \in \mathbb{R}, \chi_{1} \neq \chi_{2}$.

Hypothesis 4 (H4). The activation functions $f_{j}$ and $g_{j}, j=1,2, \ldots, m$, are bounded in $\mathbb{R}$, and $f_{j}(0)=g_{j}(0)=0, j=1,2, \ldots, m$.

Hypothesis 5 (H5). The functions $c_{i j}, w_{i j}$ and $I_{i}, i, j=1,2, \ldots, m$ are continuous on their domains.

Hypothesis 6 (H6). For any $i=1,2, \ldots, m$ and $q=1,2, \ldots, n$ there exist constants $d_{i q} \geq 0$ such that

$$
D_{i q}(t, x) \geq d_{i q}, t>0, x \in \Omega
$$


Hypothesis 7 (H7). The impulsive functions $J_{i k}$ are continuous on $\mathbb{R}$ for any $i=1, \ldots, m, k=1,2, \ldots$

Hypothesis 8 (H8). $0<t_{1}<t_{2}<\cdots<t_{k}<t_{k+1}<\ldots$, and $\lim _{k \rightarrow \infty} t_{k}=\infty$.

Remark 1. Conditions H1-H8 guarantee the existence, uniqueness and continuability of the solutions $u(t, x)=\left(u_{1}(t, x), u_{2}(t, x), \ldots, u_{m}(t, x)\right)^{T}$ of the $\operatorname{IBP}(2),(3),(4)$ on $[0, \infty) \times \Omega$ for any initial function $\varphi_{0} \in \mathcal{P} C B[30-35]$.

We will list the definitions for the stability of sets notion for impulsive reaction-diffusion delayed CGNNs. For this reason, we will need the new notations. Let $M \subset[-\tau, \infty) \times \Omega \times \mathbb{R}^{m}$.

Then, we introduce the next:

$M(t, x)=\left\{u \in \mathbb{R}^{m}:(t, x, u) \in M,(t, x) \in \mathbb{R}_{+} \times \Omega\right\} ;$

$M_{0}(t, x)=\left\{z \in \mathbb{R}^{m}:(t, x, z) \in M,(t, x) \in[-\tau, 0] \times \Omega\right\} ;$

$d(u, M(t, x))=\inf _{v \in M(t, x)}\|u-v\|_{2}$ is the distance between $u \in \mathbb{R}^{m}$ and $M(t, x)$;

$M(t, x)(\varepsilon)=\left\{u \in \mathbb{R}^{m}: d(u, M(t, x))<\varepsilon\right\}(\varepsilon>0)$ is an $\varepsilon$ - neighborhood of $M(t, x) ;$

$d_{0}\left(\varphi, M_{0}(t, x)\right)=\sup _{\xi \in[-\tau, 0]} d\left(\varphi(\xi, x), M_{0}(\xi, x)\right), \varphi \in \mathcal{P C} ;$

$M_{0}(t, x)(\varepsilon)=\left\{\varphi \in \mathcal{P C}: d_{0}\left(\varphi, M_{0}(t, x)\right)<\varepsilon\right\}$ is an $\varepsilon$ - neighborhood of $M_{0}(t, x) ;$

$\bar{M}(t, x)(\varepsilon)=\left\{u \in \mathbb{R}^{m}: d(u, M(t, x)) \leq \varepsilon\right\} ;$

$\overline{M_{0}}(t, x)(\varepsilon)=\left\{\varphi \in \mathcal{P C}: d_{0}\left(\varphi, M_{0}(t, x)\right) \leq \varepsilon\right\} ;$

$\overline{S_{\alpha}}=\left\{u \in R^{m}:\|u\|_{2} \leq \alpha\right\} ; \overline{S_{\alpha}}(\mathcal{P C})=\left\{\varphi \in \mathcal{P C}:\|\varphi\|_{\tau} \leq \alpha\right\}$, where $\|\varphi\|_{\tau}=$ $\sup _{-\tau \leq \xi \leq 0}\|\varphi(\xi, x)\|_{2}$.

We assume also that:

Hypothesis 9 (H9). For any $(t, x) \in \mathbb{R}_{+} \times \Omega$ the set $M(t, x)$ is not empty, and for any $(t, x) \in[-\tau, 0) \times \Omega$, the set $M_{0}(t, x)$ is not empty.

In our analysis, we will use the following boundedness and stability of sets definitions with respect to the impulsive control system (2).

Definition 1. The solutions of system (2) are:

(a) equi-M-bounded, if for any positive constants $\eta>0$ and $\alpha>0$ there exists a constant $\beta=\beta(\eta, \alpha)>0$ such that $x \in \Omega$ and $\varphi_{0} \in \overline{S_{\alpha}}(\mathcal{P C}) \cap \overline{M_{0}}(t, x)(\eta)$ imply

$$
u\left(t, x ; \varphi_{0}\right) \in M(t, x)(\beta), t \geq 0 ;
$$

(b) uniformly M-bounded, if the number $\beta$ from (a) depends only on $\eta$.

Definition 2. The set $M$ is said to be:

(a) uniformly stable with respect to system (2), if for any positive constants $\alpha>0$ and $\varepsilon>0$ there exists a constant $\delta=\delta(\alpha, \varepsilon)>0$ such that $x \in \Omega$ and $\varphi_{0} \in \overline{S_{\alpha}}(\mathcal{P C}) \cap M_{0}(t, x)(\delta)$ imply

$$
u\left(t, x ; \varphi_{0}\right) \in M(t, x)(\varepsilon), t \geq 0 ;
$$

(b) uniformly globally attractive with respect to system (2), if for any positive constants $\eta>0, \varepsilon>0$ and $\alpha>0$ there exists a constant $\sigma=\sigma(\eta, \varepsilon)>0$ such that $x \in \Omega$ and $\varphi_{0} \in \overline{S_{\alpha}}(\mathcal{P C}) \cap \overline{M_{0}}(t, x)(\eta)$ imply

$$
u\left(t, x ; \varphi_{0}\right) \in M(t, x)(\varepsilon), t \geq \sigma ;
$$


(c) uniformly globally asymptotically stable with respect to system (2), if $M$ is a uniformly stable and uniformly globally attractive set of system (2), and if the solutions of system (2) are uniformly M-bounded;

(d) uniformly globally exponentially stable with respect to system (2), if there exist strictly positive constants $k$ and $\lambda$ such that

$$
d\left(u\left(t, x ; \varphi_{0}\right), M(t, x)\right) \leq k d_{0}\left(\varphi_{0}, M_{0}(t, x)\right) e^{-\lambda t}, \varphi_{0} \in \mathcal{P C B}, x \in \Omega, t \geq 0
$$

Remark 2. Definition 2 is a generalization of the stability of sets definitions $[48,49,53]$ to the reaction-diffusion case.

Remark 3. Definition 2 also extends the notion of stability of a state (zero state, equilibrium state) to the stability of sets concept. For a particular choice of the set $M$ it is reduced to the existing Lyapunov-like stability definitions in the literature. For example, if $\mathbf{0}=(0,0, \ldots, 0)^{T}$ is the zero equilibrium of (2) and the set $M=[-\tau, \infty) \times \Omega \times\left\{u \in \mathbb{R}^{m}: u=\mathbf{0}\right\}$, then Definition 2 is reduced to the definition of the Lyapunov-like stability of the zero equilibrium of type (2) impulsive reaction-diffusion CGNNs .

Analogously, if $u^{*}=\left(u_{1}^{*}, u_{2}^{*}, \ldots, u_{m}^{*}\right)^{T}$ is a non-zero equilibrium of $(2)$, and the set $M=\{[-\tau, \infty) \times$ $\left.\Omega \times \mathbb{R}^{m}: u=u^{*}\right\}$, then Definition 2 is reduced to the definition of the Lyapunov-like stability of the non-zero equilibrium of the impulsive reaction-diffusion CGNNs (2) [30-35].

What follows are definitions and a comparison lemma from the Lyapunov-Razumikhin method $[15,19,28,29,42,46]$.

The main results will be proven using Lyapunov's like piecewise functions from the class $V_{0}$. For this reason, we need the sets

$$
\mathcal{G}_{k}=\left\{(t, u): t \in\left(t_{k-1}, t_{k}\right), u \in \mathbb{R}^{m}\right\}, k=1,2, \ldots, t_{0}=0, \mathcal{G}=\bigcup_{k=1}^{\infty} \mathcal{G}_{k}
$$

Definition 3. A function $V:[0, \infty) \times \mathbb{R}^{m} \rightarrow \mathbb{R}_{+}$, belongs to the class $V_{0}$ if the following conditions are fulfilled:

1. $V(t, u)$ is continuous in $\mathcal{G}$, locally Lipschitz continuous with respect to its second argument on each of the sets $\mathcal{G}_{k}$, and $V(t, u(t,))=$.0 for $(t, x, u) \in M, t \geq 0$ and $V(t, u(t,))>$.0 for $(t, x, u) \in$ $\left\{\mathbb{R}_{+} \times \Omega \times \mathbb{R}^{m}\right\} \backslash M$.

2. For each $k \in \mathbb{N}$ and $u \in \mathbb{R}^{m}$, there exist the finite limits

$$
V\left(t_{k}^{-}, u\right)=\lim _{\substack{t \rightarrow t_{k} \\ t<t_{k}}} V(t, u), \quad V\left(t_{k}^{+}, u\right)=\lim _{\substack{t \rightarrow t_{k} \\ t>t_{k}}} V(t, u),
$$

and $V\left(t_{k}^{-}, u\right)=V\left(t_{k}, u\right)$.

For a function $V \in V_{0}$ let $t \in[0, \infty), t \neq t_{k}, k=1,2, \ldots$ and $\bar{\varphi} \in \mathcal{P C}$. Then, the upper right-hand derivative of $V \in V_{0}$ with respect to the system

$$
\left\{\begin{array}{l}
\frac{d u(t, .)}{d t}=\bar{H}(t, u(t-\tau, .)), t>0, t \neq t_{k} \\
u\left(t_{k}^{+}, .\right)=u\left(t_{k} . .\right)+J_{k}\left(u\left(t_{k}, .\right)\right), k=1,2, \ldots
\end{array}\right.
$$

is defined by

$$
D^{+} V(t, \bar{\varphi}(0, .))=\lim _{\chi \rightarrow 0^{+}} \sup \frac{1}{\chi}[V(t, \bar{\varphi}(0, .))-V(t-\chi, \bar{\varphi}(0, .)-\chi \bar{H}(t, \bar{\varphi}))],
$$


where $\bar{H}:[0, \infty) \times \mathcal{P C} \rightarrow \mathbb{R}^{m}, J_{k}: \mathbb{R}^{m} \rightarrow \mathbb{R}^{m}, k=1,2, \ldots, m$,

$$
\bar{H}(t, \bar{\varphi})=\left(\bar{H}_{1}(t, \bar{\varphi}), \bar{H}_{2}(t, \bar{\varphi}), \ldots, \bar{H}_{m}(t, \bar{\varphi})\right)^{T}
$$

is continuous with respect to $(t, \bar{\varphi})$ and is locally Lipschitz continuous with respect to $\bar{\varphi} \in \mathcal{P C}$ and $J_{k}$ are continuous with respect to $u \in \mathbb{R}^{n}$.

The following comparison lemma can be proved by the same arguments used for the comparison results in $[15,28,29]$.

Lemma 1. Assume that the function $V \in V_{0}$ is such that for $t \in \mathbb{R}_{+}$and $\varphi \in \mathcal{P C}$

$$
V\left(t^{+}, \varphi(0, .)+J_{k}(\varphi),\right) \leq V(t, \varphi(0, .)), t=t_{k}, k=1,2, \ldots,
$$

and the inequality

$$
D^{+} V(t, \varphi(0, .)) \leq \mu V(t, \varphi(0, .)), t \neq t_{k}, \mu \in \mathbb{R}
$$

is valid whenever

$$
V(t+\xi, \varphi(\xi, .),) \leq V(t, \varphi(0, .)),-\tau \leq \xi \leq 0 .
$$

Then,

$$
V(t, u(t, .)) \leq \sup _{-\tau \leq \xi \leq 0} V\left(0, \varphi_{0}(\xi, .)\right) e^{\mu t}, t>0 .
$$

For the set $\Omega=\left\{x \in \mathbb{R}^{n}:\left|x_{q}\right|<l_{q}\right\}, l_{q}=$ const $>0, q=1,2, \ldots, n$, we will also need the following lemma.

Lemma 2 ([16]). Let $\Omega$ be a cube $\left|x_{q}\right|<l_{q}(q=1,2, \ldots, n)$ and let $v(x)$ be a real-valued function belonging to $C^{1}(\Omega)$, which vanishes on the boundary $\partial \Omega$ of $\Omega$, i.e., $\left.v(x)\right|_{\Omega}=0$. Then,

$$
\int_{\Omega} v^{2}(x) d x \leq l_{q}^{2} \int_{\Omega}\left|\frac{\partial v(x)}{\partial x_{q}}\right|^{2} d x .
$$

\section{Stability of Sets}

In our main theorems, we will use the Hahn class of functions $\mathcal{K}=\left\{w \in C\left[\mathbb{R}_{+}, \mathbb{R}_{+}\right]\right.$: $w$ is strictly increasing and $w(0)=0\}$.

First, we will prove a stability result for a set $M$ of a general nature.

Theorem 1. Assume that:

1. Conditions $\mathrm{H} 1-\mathrm{H} 9$ hold.

2. There exists a function $V \in V_{0}$ such that the inequalities

$$
w_{1}(d(u, M(t, x))) \leq V(t, u) \leq w_{2}(d(u, M(t, x))),
$$

hold, where $w_{1}(s) \rightarrow \infty$ as $s \rightarrow \infty,(t, x) \in \mathbb{R}_{+} \times \Omega, u \in \mathbb{R}^{m}, w_{1}, w_{2} \in \mathcal{K}$.

3. For $t \in \mathbb{R}_{+}$and $\varphi \in \mathcal{P C}$

$$
V\left(t^{+}, \varphi(0, .)+J_{k}(\varphi),\right) \leq V(t, \varphi(0, .)), t=t_{k}, k=1,2, \ldots,
$$

and the inequality

$$
D^{+} V(t, \varphi(0, .)) \leq-w_{3}(d(\varphi(0, .), M(t, x))), t \neq t_{k}, x \in \Omega
$$

is valid whenever

$$
V(t+\xi, \varphi(\xi, .),) \leq V(t, \varphi(0, .)),-\tau \leq \xi \leq 0,
$$


where $w_{3}(s)>0$ for $s>0$.

Then, the set $M$ is uniformly globally asymptotically stable with respect to system (2).

Proof. Let for arbitrary $\varepsilon>0$ we choose $\delta=\delta(\varepsilon)>0, \delta<\varepsilon$ so that $w_{1}^{-1}\left(w_{2}(\delta)\right)<\epsilon$.

For any fixed $\alpha>0$ let $\varphi_{0} \in \overline{S_{\alpha}}(\mathcal{P C}) \cap M_{0}(t, x)(\delta), x \in \Omega$ and $u(t, x)=u\left(t, x ; \varphi_{0}\right)$ be the solution of (2), (3), (4).

Then, from Lemma 1 for $\mu=0$, we get

$$
V(t, u(t, .)) \leq \sup _{-\tau \leq \xi \leq 0} V\left(0, \varphi_{0}(\xi, .)\right), t>0
$$

and from condition 2 of Theorem 1, consequently we obtain

$$
\begin{gathered}
d\left(u\left(t, x ; \varphi_{0}\right), M(t, x)\right) \leq w_{1}^{-1}\left(V(t, u(t, x)) \leq w_{1}^{-1}\left(\sup _{-\tau \leq \xi \leq 0} V\left(0, \varphi_{0}(\xi, x)\right)\right)\right. \\
\leq w_{1}^{-1}\left(w_{2}\left(d_{0}\left(\varphi_{0}, M_{0}(t, x)\right)\right)\right)<w_{1}^{-1}\left(w_{2}(\delta)\right)<\varepsilon
\end{gathered}
$$

or, $u\left(t, x ; \varphi_{0}\right) \in M(t, x)(\varepsilon)$ for all $t \geq 0$ showing that the set $M$ is uniformly stable with respect to the impulsive reaction-diffusion CGNN (2).

Next, we will prove that $u\left(t, x ; \varphi_{0}\right) \in M(t, x)(\varepsilon)$ for $t \geq \sigma$ and $\varphi_{0} \in \overline{S_{\alpha}}(\mathcal{P C}) \cap \overline{M_{0}}(t, x)(\eta), \eta>0$.

First, we will show that there exists $t^{*} \in[0, \sigma]$ such that, for any solution $u(t, x)=u\left(t, x ; \varphi_{0}\right)$ of (2) with $\varphi_{0} \in \overline{S_{\alpha}}(\mathcal{P C}) \cap \overline{M_{0}}(t, x)(\eta)$, we have

$$
d\left(u\left(t^{*}, x\right), M\left(t^{*}, x\right)\right)<\delta(\varepsilon), x \in \Omega .
$$

If we suppose that this is not true, then, for any $\sigma>0$, there exists a solution $u(t, x)=u\left(t, x ; \varphi_{0}\right)$ of (2) for which $\varphi_{0} \in \overline{S_{\alpha}}(\mathcal{P C}) \cap \overline{M_{0}}(t, x)(\eta)$, such that

$$
d(u(t, x), M(t, x)) \geq \delta(\varepsilon), t \in[0, \sigma], x \in \Omega .
$$

For $t \geq 0$ and from condition 3 of Theorem 1 , we get

$$
V(t, u(t, .))-V(0, u(0, .)) \leq \int_{0}^{t} D^{+} V(\vartheta, u(\vartheta, .)) d \vartheta \leq-\int_{0}^{t} w_{3}(d(u(\vartheta, .), M(\vartheta, .))) d \vartheta .
$$

From the other side, the properties of the function $V(t, u(t,)$.$) on \mathbb{R}_{+}$imply that it is non-increasing on the interval $[\sigma, \infty)$. Thus, the finite limit

$$
\lim _{t \rightarrow \infty} V(t, u(t, .))=v_{0} \geq 0
$$

exists.

It follows then from (9), (10) and conditions 2 of Theorem 1 that

$$
\int_{0}^{\infty} w_{3}(d(u(t, .), M(t, .))) d t \leq w_{2}(\eta)-v_{0} .
$$

From the fact that the function $w_{3}$ is strictly positive, we can conclude that the number $\sigma$ can be chosen so that

$$
\sigma>\frac{w_{2}(\eta)-v_{0}+1}{w_{3}(\delta(\varepsilon))}
$$

Then, we get

$$
w_{2}(\eta)-v_{0} \geq \int_{0}^{\infty} w_{3}(d(u(t, .), M(t, .))) d t
$$




$$
\geq \int_{0}^{\sigma} w_{3}(d(u(t, .), M(t, .))) d t \geq w_{3}(\delta(\varepsilon)) \sigma>w_{2}(\eta)-v_{0}+1,
$$

which is a contradiction.

Thus, there exist a positive constant $\sigma=\sigma(\epsilon, \eta)$ and a $t^{*} \in[0, \sigma]$ such that, for any solution $u(t, x)=u\left(t, x ; \varphi_{0}\right)$ of the impulsive reaction-diffusion CGNN (2) such that $\varphi_{0} \in \overline{S_{\alpha}}(\mathcal{P C}) \cap \overline{M_{0}}(t, x)(\eta)$, the inequality $(7)$ holds.

Therefore, for $t \geq t^{*}$ (hence for any $t \geq \sigma$ as well) and $x \in \Omega$, we have

$$
\begin{gathered}
d(u(t, x), M(t, x)) \leq w_{1}^{-1}(V(t, u(t, x))) \leq w_{1}^{-1}\left(V\left(t^{*}, u\left(t^{*}, x\right)\right)\right) \\
\leq w_{1}^{-1}\left(w_{2}\left(d\left(u\left(t^{*}, x\right), M\left(t^{*}, x\right)\right)\right)\right)<w_{1}^{-1}\left(w_{2}(\delta)\right)<\epsilon,
\end{gathered}
$$

which proves that the set $M$ is uniformly globally attractive with respect to the impulsive reaction-diffusion CGNN (2).

Finally, we will prove that the solutions of (2) are uniformly $M$-bounded. Let $\eta>0$ and choose the number $\beta=\beta(\eta)>0$ so that $w_{1}^{-1}\left(w_{2}(\eta)\right)<\beta, \beta>\eta$.

For any fixed number $\alpha>0$ and for $\varphi_{0} \in \overline{S_{\alpha}}(\mathcal{P C}) \cap \overline{M_{0}}(t, x)(\eta)$, by (6) and condition 2 of Theorem 1, we obtain

$$
\begin{gathered}
d\left(u\left(t, x ; \varphi_{0}\right), M(t, x)\right) \leq w_{1}^{-1}\left(V(t, u(t, x)) \leq w_{1}^{-1}\left(\sup _{-\tau \leq \xi \leq 0} V\left(0, \varphi_{0}(\xi, x)\right)\right)\right. \\
\leq w_{1}^{-1}\left(w_{2}\left(d_{0}\left(\varphi_{0}, M_{0}(t, x)\right)\right)\right)<w_{1}^{-1}\left(w_{2}(\eta)\right)<\beta
\end{gathered}
$$

for $t \in \mathbb{R}_{+}$. Hence, $u\left(t, x ; \varphi_{0}\right) \in M(t, x)(\beta)$ for $t \geq 0$.

The proof of Theorem 1 is complete.

Theorem 2. If, in Theorem 1, $w_{i}=c_{i} d^{r}(u, M(t, x))$ for $(t, x) \in \mathbb{R}_{+} \times \Omega, u \in \mathbb{R}^{m}, i=1,2$, and $w_{3}=$ $\left.c_{3} d^{r}(\varphi(0,),. M(t, x))\right)$ for $t \neq t_{k}, x \in \Omega, \varphi \in \mathcal{P C}$, where $c_{i}>0$ are constants $i=1,2,3, r \geq 1$, then the set $M$ is uniformly globally exponentially stable with respect to system (2).

Proof. Let $\varphi_{0} \in \mathcal{P C B}$ and $u(t, x)=u\left(t, x ; \varphi_{0}\right)$ be the solution of (2), (3), (4).

For $w_{i}=c_{i} d^{r}(u, M(t, x))$ for $(t, x) \in \mathbb{R}_{+} \times \Omega, u \in \mathbb{R}^{m}, i=1,2$, and $\left.w_{3}=c_{3} d^{r}(\varphi(0,),. M(t, x))\right)$ for $t \neq t_{k}, x \in \Omega, \varphi \in \mathcal{P C}$, where $c_{i}>0$ are constants $i=1,2,3, r \geq 1$, we have that the inequality

$$
D^{+} V(t, \varphi(0, .)) \leq-\frac{c_{3}}{c_{2}} V(t, \varphi(0, .)), t \neq t_{k}
$$

is valid whenever

$$
V(t+\xi, \varphi(\xi, .),) \leq V(t, \varphi(0, .)),-\tau \leq \xi \leq 0, \varphi \in \mathcal{P C} .
$$

Hence, in view of Lemma 1, we have

$$
V(t, u(t, .)) \leq \sup _{-\tau \leq \xi \leq 0} V\left(0, \varphi_{0}(\xi, .)\right) e^{-\frac{c_{3}}{c_{2}} t}, t>0
$$

and, therefore,

$$
\begin{aligned}
d(u(t, x), M(t, x)) \leq & \left(\frac{1}{c_{1}} V(t, u(t, x))\right)^{1 / r} \leq\left(\frac{1}{c_{1}} \sup _{-\tau \leq \xi \leq 0} V\left(0, \varphi_{0}(\xi, .)\right) e^{-\frac{c_{3}}{c_{2}} t}\right)^{1 / r} \\
& \leq\left(\frac{c_{2}}{c_{1}}\right)^{1 / r} d_{0}\left(\varphi_{0}, M_{0}(t, x)\right)^{-\frac{c_{3}}{r_{2}} t}, t \geq 0,
\end{aligned}
$$

which proves that the set $M$ is uniformly globally exponentially stable with respect to system (2). 
Remark 4. Theorems 1 and 2 offer sufficient conditions for the more general concept of stability of sets for the impulsive reaction-diffusion CGCN model (2). The set M considered in the theorems is of a very general nature. The obtained results are important in the case when the corresponding sets are attractors of type (2) models. For a particular choice of the set $M$, they include results for the following specific cases:

(i) If $M=\left\{[-\tau, \infty) \times \Omega \times \mathbb{R}^{m}: u=u^{*}\right\}$, where $u^{*}$ is an equilibrium state of $(2)$, then $d(u, M)=$ $\inf \left\{\|u-v\|_{2}: v=u^{*}\right\}=\|u-v\|_{2} ;$

(ii) If $M=M_{R}=\left\{u \in \mathbb{R}^{m}:\|u\|_{2} \leq R\right\}$, then $d(u, M)=\max \left\{0,\|u\|_{2}-R\right\}$.

Thus, our set-stability results are extensions to the existing stability theory for impulsive reaction-diffusion CGNNs [30-35].

In the next result, we will consider a specific attractor set $M$. Let the set $\Omega$ of points $x, x=$ $\left(x_{1}, x_{2}, \ldots, x_{n}\right)^{T}$ is such that $\left|x_{q}\right|<l_{q}$, where $l_{q}, q=1,2 \ldots, n$, are positive constants.

Set $\tilde{d}_{i}=\sum_{q=1}^{n} \frac{d_{i q}}{l_{q}^{2}}, i=1,2, \ldots, m, c_{i j}^{+}=\sup _{t \in \mathbb{R}_{+}} c_{i j}(t), w_{i j}^{+}=\sup _{t \in \mathbb{R}_{+}} w_{i j}(t)$.

Let $\underline{u}^{*}=\left(\underline{u}_{1}^{*}, \underline{u}_{2}^{*}, \ldots, \underline{u}_{m}^{*}\right)^{T} \in \mathbb{R}_{+}^{m}$ and $\bar{u}^{*}=\left(\bar{u}_{1}^{*}, \bar{u}_{2}^{*}, . ., \bar{u}_{m}^{*}\right)^{T} \in \mathbb{R}_{+}^{m}$, where $\underline{u}_{i}^{*}=\left\{\underline{u}_{i k}^{*}\right\}, \bar{u}_{i}^{*}=$ $\left\{\bar{u}_{i k}^{*}\right\}, i=1,2, \ldots, m, k=1,2, \ldots$ be two constant solutions of the reaction-diffusion CGNN (2), i.e.,

$$
\left\{\begin{array}{l}
0=-a_{i}\left(\underline{u}_{i}^{*}(t, x)\right)\left[b_{i}\left(\underline{u}_{i}^{*}(t, x)\right)-I_{i}(t, x)-\sum_{j=1}^{m} c_{i j}(t) f_{j}\left(\underline{u}_{j}^{*}(t, x)\right)\right. \\
\left.\quad-\sum_{j=1}^{m} w_{i j}(t) g_{j}\left(\underline{u}_{j}^{*}\left(t-\tau_{j}(t), x\right)\right)\right], t \neq t_{k}, \\
\underline{u}_{i}^{*}\left(t_{k}^{+}, x\right)=\underline{u}_{i}^{*}\left(t_{k}, x\right),
\end{array}\right.
$$

and

$$
\left\{\begin{array}{l}
0=-a_{i}\left(\bar{u}_{i}^{*}(t, x)\right)\left[b_{i}\left(\bar{u}_{i}^{*}(t, x)\right)-I_{i}(t, x)-\sum_{j=1}^{m} c_{i j}(t) f_{j}\left(\bar{u}_{j}^{*}(t, x)\right)\right. \\
\left.\quad-\sum_{j=1}^{m} w_{i j}(t) g_{j}\left(\bar{u}_{j}^{*}\left(t-\tau_{j}(t), x\right)\right)\right], t \neq t_{k}, \\
\bar{u}_{i}^{*}\left(t_{k}^{+}, x\right)=\bar{u}_{i}^{*}\left(t_{k}, x\right) .
\end{array}\right.
$$

Theorem 3. Assume that:

1. Conditions H1-H9 hold.

2. The following condition met

$$
\begin{gathered}
\min _{1 \leq i \leq m}\left[2\left(\tilde{d}_{i}+\underline{a}_{i} B_{i}\right)-\bar{a}_{i} \sum_{j=1}^{m}\left(L_{j} c_{i j}^{+}+M_{j} w_{i j}^{+}+L_{i} c_{j i}^{+}\right)\right] \\
>\max _{1 \leq i \leq m}\left(M_{i} \sum_{j=1}^{m} \bar{a}_{j} w_{j i}^{+}\right)>0 .
\end{gathered}
$$

3. The functions $J_{i k}$ are such that

$$
J_{i k}\left(u_{i}\left(t_{k}, x\right)\right)=-\gamma_{i k} u_{i}\left(t_{k}, x\right), \quad 0<\gamma_{i k}<2
$$

$i=1,2, \ldots, m, k=1,2, \ldots$.

Then, the set $M=[-\tau, \infty) \times \Omega \times\left\{\mathbb{R}^{m}: \underline{u}_{i}^{*} \leq u_{i} \leq \bar{u}_{i}^{*}, i=1,2, \ldots, m\right\}$ is uniformly globally exponentially stable with respect to the impulsive reaction-diffusion CGNN (2). 
Proof. Let $\varphi_{0} \in \mathcal{P C B}, \varphi_{0}=\left(\varphi_{01}, \varphi_{02}, \ldots, \varphi_{0 m}\right)^{T}$ and $u(t, x)=u\left(t, x ; \varphi_{0}\right), u(t, x)=$ $\left(u_{1}(t, x), u_{2}(t, x), \ldots, u_{m}(t, x)\right)^{T}$ be the solution of the CGNN (2) with initial function $\varphi_{0}$.

For $v \in M(t, x), v=v(t, x)=\left(v_{1}(t, x), v_{2}(t, x), \ldots, v_{m}(t, x)\right)^{T},(t, x) \in[-\tau, \infty) \times \Omega$, we consider the Lyapunov candidate function

$$
V(u(t, .), v(t, .))=\frac{1}{2}\left(\inf _{v \in M(t, x)}\|u-v\|_{2}\right)^{2}=\frac{1}{2} d^{2}(u, M(t, x)) .
$$

For $t \geq 0$ and $t=t_{k}, k=1,2, \ldots$, from condition 3 of the theorem, we obtain

$$
\frac{1}{2} \int_{\Omega} \sum_{i=1}^{m}\left(1-\gamma_{i k}\right)^{2}\left(u_{i}\left(t_{k}, x\right)-v_{i}(t, x)\right)^{2} d x<\frac{1}{2} \int_{\Omega} \sum_{i=1}^{m}\left(u_{i}\left(t_{k}, x\right)-v_{i}(t, x)\right)^{2} d x
$$

and hence

$$
V\left(u\left(t_{k}^{+}, .\right), v\left(t_{k}^{+}, .\right)\right)<V\left(u\left(t_{k}, .\right), v_{k}(t, .)\right) .
$$

In addition, for $t \geq t_{0}$ and $t \in\left(t_{k-1}, t_{k}\right], k=1,2, \ldots$, we have that

$$
\frac{1}{2} \frac{d}{d t} \| u(t, .)-v\left(t, . \|_{2}^{2} \leq \int_{\Omega} \sum_{i=1}^{m}\left|u_{i}(t, x)-v_{i}(t, x)\right| \frac{\partial\left(u_{i}(t, x)-v_{i}(t, x)\right)}{\partial t} d x .\right.
$$

First, we will consider the case, when $u_{i}(t, x) \geq v_{i}(t, x)$ for any $i=1,2, \ldots, m$ and $(t, x) \in$ $[-\tau, \infty) \times \Omega$.

From the choice of the set $M$, we get

$$
\frac{1}{2} \frac{d}{d t}\|u(t, .)-v(t, .)\|_{2}^{2} \leq \sum_{i=1}^{m} \int_{\Omega}\left(u_{i}(t, x)-\underline{u}_{i}^{*}\right) \frac{\partial\left(u_{i}(t, x)-\underline{u}_{i}^{*}\right)}{\partial t} d x .
$$

Since $\underline{u}^{*}=\left(\underline{u}_{1}^{*}, \underline{u}_{2}^{*}, \ldots, \underline{u}_{m}^{*}\right)^{T}$ is a constant solution of (2), then, by H1, we obtain

$$
\begin{aligned}
& \left(u_{i}(t, x)-\underline{u}_{i}^{*}\right) \frac{\partial\left(u_{i}(t, x)-\underline{u}_{i}^{*}\right)}{\partial t} \leq\left(u_{i}(t, x)-\underline{u}_{i}^{*}\right)\left(\sum_{q=1}^{n} \frac{\partial}{\partial x_{q}}\left(D_{i q} \frac{\partial\left(u_{i}(t, x)-\underline{u}_{i}^{*}\right)}{\partial x_{q}}\right)\right. \\
& -\underline{a}_{i}\left[b_{i}\left(u_{i}(t, x)\right)-b_{i}\left(\underline{u}_{i}^{*}\right)\right]+\bar{a}_{i} \sum_{j=1}^{m} c_{i j}^{+}\left|f_{j}\left(u_{j}(t, x)\right)-f_{j}\left(\underline{u}_{j}^{*}\right)\right| \\
& \left.+\bar{a}_{i} \sum_{j=1}^{m} w_{i j}^{+}\left|g_{j}\left(u_{j}\left(t-\tau_{j}(t), x\right)\right)-g_{j}\left(\underline{u}_{j}^{*}\right)\right|\right) .
\end{aligned}
$$

Then, we integrate (15) over $\Omega$ and obtain

$$
\begin{aligned}
& \int_{\Omega}\left(u_{i}(t, x)-\underline{u}_{i}^{*}\right) \frac{\partial\left(u_{i}(t, x)-\underline{u}_{i}^{*}\right)}{\partial t} \leq \int_{\Omega} \sum_{q=1}^{n} \frac{\partial}{\partial x_{q}}\left(D_{i q} \frac{\partial\left(u_{i}(t, x)-\underline{u}_{i}^{*}\right)}{\partial x_{q}}\right)\left(u_{i}(t, x)-\underline{u}_{i}^{*}\right) d x \\
& -\int_{\Omega} \underline{a}_{i}\left(u_{i}(t, x)-\underline{u}_{i}^{*}\right)\left[b_{i}\left(u_{i}(t, x)\right)-b_{i}\left(\underline{u}_{i}^{*}\right)\right] d x \\
& +\bar{a}_{i} \int_{\Omega}\left(u_{i}(t, x)-\underline{u}_{i}^{*}\right) \sum_{j=1}^{m} c_{i j}^{+}\left|f_{j}\left(u_{j}(t, x)\right)-f_{j}\left(\underline{u}_{j}^{*}\right)\right| d x \\
& +\bar{a}_{i} \int_{\Omega}\left(u_{i}(t, x)-\underline{u}_{i}^{*}\right) \sum_{j=1}^{m} w_{i j}^{+}\left|g_{j}\left(u_{j}\left(t-\tau_{j}(t), x\right)\right)-g_{j}\left(\underline{u}_{j}^{*}\right)\right| d x .
\end{aligned}
$$


Next, from the Dirichlet-type boundary conditions, H6 and Lemma 2, by the Green's theorem, we have

$$
\begin{aligned}
& \int_{\Omega} \sum_{q=1}^{n} \frac{\partial}{\partial x_{q}}\left(D_{i q} \frac{\partial\left(u_{i}(t, x)-\underline{u}_{i}^{*}\right)}{\partial x_{q}}\right)\left(u_{i}(t, x)-\underline{u}_{i}^{*}\right) d x \\
& =-\sum_{q=1}^{n} \int_{\Omega} D_{i q}\left(\frac{\partial\left(u_{i}(t, x)-\underline{u}_{i}^{*}\right)}{\partial x_{q}}\right)^{2} d x \\
& \leq-\sum_{q=1}^{n} \int_{\Omega} d_{i q}\left(\frac{\partial\left(u_{i}(t, x)-\underline{u}_{i}^{*}\right)}{\partial x_{q}}\right)^{2} d x \\
& \leq-\sum_{q=1}^{n} \int_{\Omega} \frac{d_{i q}}{l_{q}^{2}}\left(u_{i}(t, x)-\underline{u}_{i}^{*}\right)^{2} d x=-\tilde{d}_{i} \int_{\Omega}\left(u_{i}(t, x)-\underline{u}_{i}^{*}\right)^{2} d x .
\end{aligned}
$$

\section{Conditions H1-H4 imply}

$$
\int_{\Omega} \underline{a}_{i}\left(u_{i}(t, x)-\underline{u}_{i}^{*}\right)\left[b_{i}\left(u_{i}(t, x)\right)-b_{i}\left(\underline{u}_{i}^{*}\right)\right] d x \geq \underline{a}_{i} B_{i} \int_{\Omega}\left(u_{i}(t, x)-\underline{u}_{i}^{*}\right)^{2} d x,
$$

and

$$
\begin{aligned}
& \bar{a}_{i} \int_{\Omega}\left(u_{i}(t, x)-\underline{u}_{i}^{*}\right) \sum_{j=1}^{m} c_{i j}^{+}\left|f_{j}\left(u_{j}(t, x)\right)-f_{j}\left(\underline{u}_{j}^{*}\right)\right| d x \\
& \leq \bar{a}_{i} \int_{\Omega} \sum_{j=1}^{m} c_{i j}^{+} L_{j}\left|u_{i}(t, x)-\underline{u}_{i}^{*}\right|\left|u_{j}(t, x)-\underline{u}_{j}^{*}\right| d x \\
& \leq \frac{1}{2} \bar{a}_{i} \sum_{j=1}^{m} \int_{\Omega} c_{i j}^{+} L_{j}\left[\left(u_{i}(t, x)-\underline{u}_{i}^{*}\right)^{2}+\left(u_{j}(t, x)-\underline{u}_{j}^{*}\right)^{2}\right] d x .
\end{aligned}
$$

In addition,

$$
\begin{aligned}
& \bar{a}_{i} \int_{\Omega}\left(u_{i}(t, x)-\underline{u}_{i}^{*}\right) \sum_{j=1}^{m} w_{i j}^{+}\left|g_{j}\left(u_{j}\left(t-\tau_{j}(t), x\right)\right)-g_{j}\left(\underline{u}_{j}^{*}\right)\right| d x \\
& \leq \bar{a}_{i} \sum_{j=1}^{m} \int_{\Omega} w_{i j}^{+} M_{j}\left|u_{i}(t, x)-\underline{u}_{i}^{*}\right|\left|u_{j}\left(t-\tau_{j}(t), x\right)-\underline{u}_{j}^{*}\right| d x \\
& \leq \frac{1}{2} \bar{a}_{i} \sum_{j=1}^{m} \int_{\Omega} w_{i j}^{+} M_{j}\left[\left(u_{i}(t, x)-\underline{u}_{i}^{*}\right)^{2}+\left(u_{j}\left(t-\tau_{j}(t), x\right)-\underline{u}_{j}^{*}\right)^{2}\right] d x .
\end{aligned}
$$


In view of (16)-(20), we have

$$
\begin{aligned}
& \frac{1}{2} \frac{d}{d t}\|u(t, .)-v(t, .)\|_{2}^{2} \\
& \leq \sum_{i=1}^{m}\left[-\left(\tilde{d}_{i}+\underline{a}_{i} B_{i}\right) \int_{\Omega}\left(u_{i}(t, x)-\underline{u}_{i}^{*}\right)^{2} d x\right. \\
& +\frac{1}{2} \bar{a}_{i} \sum_{j=1}^{m} \int_{\Omega} c_{i j}^{+} L_{j}\left[\left(u_{i}(t, x)-\underline{u}_{i}^{*}\right)^{2}+\left(u_{j}(t, x)-\underline{u}_{j}^{*}\right)^{2}\right] d x \\
& \left.\left.+\frac{1}{2} \bar{a}_{i} \sum_{j=1}^{m} \int_{\Omega} w_{i j}^{+} M_{j}\left[\left(u_{i}(t, x)-\underline{u}_{i}^{*}\right)^{2}+\left(u_{j}\left(t-\tau_{j}(t), x\right)-\underline{u}_{j}^{*}\right)^{2}\right] d x\right]\right] \\
& \leq-\frac{1}{2} \sum_{i=1}^{m}\left[2\left(\tilde{d}_{i}+\underline{a}_{i} B_{i}\right)\right. \\
& \left.-\bar{a}_{i} \sum_{j=1}^{m}\left(L_{j} c_{i j}^{+}+M_{j} w_{i j}^{+}+L_{i} c_{j i}^{+}\right)\right] \int_{\Omega}\left(u_{i}(t, x)-\underline{u}_{i}^{*}\right)^{2} d x \\
& +\frac{1}{2} \sum_{i=1}^{m} \sum_{j=1}^{m} \bar{a}_{j} w_{j i}^{+} M_{i} \int_{\Omega} \sup _{-\tau \leq \tilde{\xi} \leq 0}\left(u_{j}\left(\xi_{,} x\right)-\underline{u}_{j}^{*}\right)^{2} d x \\
& \leq \max _{1 \leq i \leq m}\left(M_{i} \sum_{j=1}^{m} \bar{a}_{j} w_{j i}^{+}\right) \frac{1}{2}\left\|u-\underline{u}^{*}\right\|_{\tau}^{2}=-c_{1} \frac{1}{2}\left\|u(t, .)-\underline{u}^{*}\right\|_{2}^{2}+c_{2} \frac{1}{2}\left\|u-\underline{u}^{*}\right\|_{\tau}^{2}, \\
& \left.\leq 2\left(\tilde{d}_{i}+\underline{a}_{i} B_{i}\right)-\bar{a}_{i} \sum_{j=1}\left(L_{j} c_{i j}^{+}+M_{j} w_{i j}^{+}+L_{i} c_{j i}^{+}\right)\right] \frac{1}{2}\left\|u(t, .)-\underline{u}^{*}\right\|_{2}^{2}
\end{aligned}
$$

where

$$
\begin{gathered}
c_{1}=\min _{1 \leq i \leq m}\left[2\left(\tilde{d}_{i}+\underline{a}_{i} B_{i}\right)-\bar{a}_{i} \sum_{j=1}^{m}\left(L_{j} c_{i j}^{+}+M_{j} w_{i j}^{+}+L_{i} c_{j i}^{+}\right)\right], \\
c_{2}=\max _{1 \leq i \leq m}\left(M_{i} \sum_{j=1}^{m} \bar{a}_{j} w_{j i}^{+}\right) .
\end{gathered}
$$

From condition 2 of Theorem 3, it follows that there exists a constant $c>0$ such that

$$
\frac{1}{2} \frac{d}{d t}\|u(t, .)-v(t, .)\|_{2}^{2} \leq-\frac{1}{2} c\|u(t, .)-v(t, .)\|_{2}^{2} .
$$

By repeating the same arguments, we can conduct the proof of the inequality (22) in the case when $u_{i}(t, x)<v_{i}(t, x)$ for any $i=1,2, \ldots, m$ and $(t, x) \in[-\tau, \infty) \times \Omega$, and, in any other case, when $u_{i}(t, x)<v_{i}(t, x)$ for some $i=1,2, \ldots, m$ and $u_{i}(t, x) \geq v_{i}(t, x)$ for the rest of the variables of $u(t, x)$ and $v(t, x),(t, x) \in[-\tau, \infty) \times \Omega$.

Using (22) for the upper right-hand derivative of $V$ along the solutions of system (2), for $\tilde{\varphi} \in \mathcal{C}$, $\tilde{\varphi}=\left(\tilde{\varphi}_{1}, \tilde{\varphi}_{2}, \ldots, \tilde{\varphi}_{m}\right)^{T}$, we have

$$
D^{+} V(t, \tilde{\varphi}(0, .)) \leq-c V(t, \tilde{\varphi}(0, .))
$$

when $V(t+\xi, \tilde{\varphi}(\xi,).) \leq V(t, \tilde{\varphi}(0,)$.$) for -\tau \leq \xi \leq 0, \tilde{\varphi} \in \mathcal{P C}, t \geq 0$.

Now, applying Theorem 2 , we conclude that the set $M=[-\tau, \infty) \times \Omega \times\left\{\mathbb{R}^{m}: \underline{u}_{i}^{*} \leq u_{i} \leq \bar{u}_{i}^{*}, i=\right.$ $1,2, \ldots, m\}$ is uniformly globally exponentially stable with respect to the impulsive reaction-diffusion CGNN (2). 


\section{Robust Stability of Sets}

In this section, a robust stability of sets result for the impulsive reaction-diffusion CGNN model (2) will be presented. To this end, we will extend the model (2) to incorporate uncertain terms.

Consider the following uncertain impulsive reaction-diffusion delayed CGNN corresponding to the system (2)

$$
\left\{\begin{aligned}
& \frac{\partial u_{i}(t, x)}{\partial t}=\sum_{q=1}^{n} \frac{\partial}{\partial x_{q}}\left(D_{i q} \frac{\partial u_{i}(t, x)}{\partial x_{q}}\right) \\
&-\left(a_{i}\left(u_{i}(t, x)\right)+\tilde{a}_{i}\left(u_{i}(t, x)\right)\right)\left[\left(b_{i}\left(u_{i}(t, x)\right)+\tilde{b}_{i}\left(u_{i}(t, x)\right)\right)\right. \\
&-I_{i}(t, x)-\tilde{I}_{i}(t, x) \\
&-\sum_{j=1}^{m}\left(c_{i j}(t)+\tilde{c}_{i j}(t)\right)\left(f_{j}\left(u_{j}(t, x)\right)+\tilde{f}_{j}\left(u_{j}(t, x)\right)\right) \\
&\left.-\sum_{j=1}^{m}\left(w_{i j}(t)+\tilde{w}_{i j}(t)\right)\left(g_{j}\left(u_{j}\left(t-\tau_{j}(t), x\right)\right)+\tilde{g}_{j}\left(u_{j}\left(t-\tau_{j}(t), x\right)\right)\right)\right], t \neq t_{k}, \\
& u_{i}\left(t_{k}+, .\right)-u\left(t_{k} .\right)=-\left(\gamma_{i k}+\tilde{\gamma}_{i k}\right) u_{i}\left(t_{k}, x\right),
\end{aligned}\right.
$$

where $\tilde{a}_{i}, \tilde{b}_{i}, \tilde{c}_{i j}, \tilde{w}_{i j}, \tilde{f}_{j}, \tilde{g}_{j}, \tilde{I}_{i}, \tilde{\gamma}_{i k}, i, j=1, \ldots, m, k=1,2, \ldots$ are all continuous functions in their domains, and represent the uncertain terms in the system (23) [54-57]. Note that, if all of these functions are zeros, then we will recover the "nominal system" (2) [54].

In numerous applications, the activation function of a neural network model may involve uncertain terms. Uncertain parameters also appeared in the connection coefficients as well as in the external inputs, due to uncertainty in the environment, data measurement, etc. See, for example, $[5,7,21,56,57]$ and the references therein. Thus, it is essential to study the effect of uncertain terms on the stability behavior of impulsive reaction-diffusion CGNNs.

With the next definition, we introduce the notion of robust exponential stability of a set with respect to system (2).

Definition 4. The set $M$ is said to be robustly uniformly globally exponentially stable with respect to system (2) if for any functions $\tilde{a}_{i}, \tilde{b}_{i}, \tilde{c}_{i j}, \tilde{w}_{i j}, \tilde{f}_{j}, \tilde{g}_{j}, \tilde{I}_{i}, i, j=1, \ldots, m$, the set $M$ is uniformly globally exponentially stable with respect to system (23).

Introduce the following conditions:

Hypothesis 10 (H10). For $\tilde{a}_{i}^{+}=\sup _{\chi \in \mathbb{R}} \tilde{a}_{i}(\chi), i=1,2, \ldots, m$, we have

$$
\tilde{a}_{i}^{+} \in\left[\underline{a}_{i}-a_{i}, \bar{a}_{i}-a_{i}\right] .
$$

Hypothesis 11 (H11). The functions $\tilde{b}_{i}, i=1,2, \ldots, m$, are continuous and

$$
\frac{b_{i}\left(\chi_{1}\right)+\tilde{b}_{i}\left(\chi_{1}\right)-\left(b_{i}\left(\chi_{2}\right)+\tilde{b}_{i}\left(\chi_{2}\right)\right)}{\chi_{1}-\chi_{2}} \geq B_{i}
$$


for $\chi_{1}, \chi_{2} \in \mathbb{R}, \chi_{1} \neq \chi_{2}$.

Hypothesis 12 (H12). The functions $\tilde{c}_{i j}, \tilde{I}_{i}, \tilde{w}_{i j}, i, j=1,2, \ldots, m$, are continuous on their domains, and

$$
\sup _{t \in \mathbb{R}} \tilde{c}_{i j}(t)=\tilde{c}_{i j}^{+}, \sup _{t \in \mathbb{R}} \tilde{w}_{i j}(t)=\tilde{w}_{i j}^{+} .
$$

Hypothesis 13 (H13). There exist positive constants $\tilde{L}_{i}, \tilde{M}_{i}, i=1,2, \ldots, m$, with

$$
\begin{aligned}
& \left|\tilde{f}_{i}\left(\chi_{1}\right)-\tilde{f}_{i}\left(\chi_{2}\right)\right| \leq \tilde{L}_{i}\left|\chi_{1}-\chi_{2}\right|, \\
& \left|\tilde{g}_{i}\left(\chi_{1}\right)-\tilde{g}_{i}\left(\chi_{2}\right) \leq \tilde{M}_{i}\right| \chi_{1}-\chi_{2} \mid
\end{aligned}
$$

for all $\chi_{1}, \chi_{2} \in \mathbb{R}, \chi_{1} \neq \chi_{2}$, and $\tilde{f}_{i}(0)=\tilde{g}_{i}(0)=0$.

Hypothesis 14 (H14). The unknown constants $\tilde{\gamma}_{i k}$ are bounded and $\tilde{\gamma}_{i k} \in\left[-1-\gamma_{i k}, 1-\gamma_{i k}\right], i=1,2, \ldots, m$.

Theorem 4. Assume that

1. Conditions $\mathrm{H} 1-\mathrm{H} 14$ hold.

2. The inequality

$$
\begin{gathered}
\min _{1 \leq i \leq m}\left[2\left(\tilde{d}_{i}+\underline{a}_{i} B_{i}\right)\right. \\
\left.-\bar{a}_{i} \sum_{j=1}^{m}\left(\left(L_{j}+\tilde{L}_{j}\right)\left(c_{i j}^{+}+\tilde{c}_{i j}^{+}\right)+\left(M_{j}+\tilde{M}_{j}\right)\left(w_{i j}^{+}+\tilde{w}_{i j}^{+}\right)+\left(L_{i}+\tilde{L}_{i}\right)\left(c_{j i}^{+}+\tilde{c}_{j i}^{+}\right)\right)\right] \\
>\max _{1 \leq i \leq m}\left(\left(M_{i}+\tilde{M}_{i}\right) \sum_{j=1}^{m} \bar{a}_{j} w_{j i}^{+}\right)>0
\end{gathered}
$$

is satisfied.

Then, the set $M=[-\tau, \infty) \times \Omega \times\left\{\mathbb{R}^{m}: \underline{u}_{i}^{*} \leq u_{i} \leq \bar{u}_{i}^{*}, i=1,2, \ldots, m\right\}$ is robustly uniformly globally exponentially stable with respect to the impulsive reaction-diffusion CGNN (2).

Proof. The proof of the uniform global exponential stability of the set $M$ with respect to system (23) for any values of the uncertain terms can be conducted similarly to the proof of Theorem 3 using the assumptions H1-H14. Hence, it follows by Definition 4 that the set $M=[-\tau, \infty) \times \Omega \times\left\{\mathbb{R}^{m}\right.$ : $\left.\underline{u}_{i}^{*} \leq u_{i} \leq \bar{u}_{i}^{*}, i=1,2, \ldots, m\right\}$ is robustly uniformly globally exponentially stable with respect to the impulsive reaction-diffusion CGNN (2).

\section{Examples}

In this section, the effectiveness of the proposed sufficient conditions is demonstrated through two examples. 
Example 1. Consider an impulsive reaction-diffusion CGNN model (2), where $\Omega=\left\{x \in \mathbb{R}^{2}:\left|x_{q}\right|<1, q=\right.$ $1,2\}$ given by

$$
\left\{\begin{aligned}
& \frac{\partial u_{i}(t, x)}{\partial t}= \sum_{q=1}^{2} \frac{\partial}{\partial x_{q}}\left(D_{i q} \frac{\partial u_{i}(t, x)}{\partial x_{q}}\right)-a_{i}\left(u_{i}(t, x)\right)\left[b_{i}\left(u_{i}(t, x)\right)\right. \\
&-I_{i}(t, x)-\sum_{j=1}^{2} c_{i j}(t) f_{j}\left(u_{j}(t, x)\right) \\
&\left.-\sum_{j=1}^{2} w_{i j}(t) g_{j}\left(u_{j}\left(t-\tau_{j}(t), x\right)\right)\right], t \neq t_{k}, k=1,2, \ldots, \\
& u\left(t_{k}^{+}, x\right)- u\left(t_{k}, x\right)=\left(\begin{array}{lr}
-2 / 5 & 0 \\
0 & -1 / 7
\end{array}\right) u\left(t_{k}, x\right), k=1,2, \ldots, \\
& u_{i}(t, x)=0, t \in[-\tau, \infty), x \in \partial \Omega, \\
& u_{i}(s, x)=\varphi_{0 i}(s, x), s \in[-\tau, 0], x \in \Omega,
\end{aligned}\right.
$$

where $t>0,0<t_{1}<t_{2}<\cdots<t_{k}<t_{k+1}<\ldots, \lim _{k \rightarrow \infty} t_{k}=\infty, I_{1}=I_{2}=0, f_{i}\left(u_{i}\right)=g_{i}\left(u_{i}\right)=$ $\frac{1}{2}\left(\left|u_{i}+1\right|-\left|u_{i}-1\right|\right), \tau_{1}(t)=\tau_{2}(t)=e^{t} /\left(1+e^{t}\right), 0 \leq \tau_{i}(t) \leq \tau(\tau=1), a_{i}\left(u_{i}\right)=1, b_{1}\left(u_{i}\right)=u_{i}$, $b_{2}\left(u_{i}\right)=3 u_{i}, i=1,2$,

$$
\begin{aligned}
& \left(c_{i j}\right)_{2 \times 2}(t)=\left(\begin{array}{ll}
c_{11}(t) & c_{12}(t) \\
c_{21}(t) & c_{22}(t)
\end{array}\right)=\left(\begin{array}{cc}
0.6-0.4 \sin (t) & 0.1-0.4 \cos (t) \\
0.2-0.4 \cos (t) & 0.2-0.3 \sin (t)
\end{array}\right), \\
& \left(w_{i j}\right)_{2 \times 2}(t)=\left(\begin{array}{cc}
w_{11}(t) & w_{12}(t) \\
w_{21}(t) & w_{22}(t)
\end{array}\right)=\left(\begin{array}{cc}
0.3 \sin (t) & 0.4 \cos (t) \\
0.4 \cos (t) & 0.6 \sin (t)
\end{array}\right), \\
& \left(D_{i k}\right)_{2 \times 2}=\left(\begin{array}{ll}
D_{11} & D_{12} \\
D_{21} & D_{22}
\end{array}\right)=\left(\begin{array}{lr}
1+2 \sin t & 0 \\
0 & \cos t
\end{array}\right) .
\end{aligned}
$$

Clearly, we have that the assumptions H1-H9 are satisfied for $\underline{a}_{i}=\bar{a}_{i}=1, i=1,2, B_{1}=1, B_{2}=3$, $L_{1}=L_{2}=M_{1}=M_{2}=1$ and

$$
\left(d_{i k}\right)_{2 \times 2}=\left(\begin{array}{ll}
d_{11} & d_{12} \\
d_{21} & d_{22}
\end{array}\right)=\left(\begin{array}{cc}
3 & 0 \\
0 & 1
\end{array}\right) .
$$

Conditions H1-H9 also guarantee the existence of a unique equilibrium point $u^{*}=\left(u_{1}^{*}, u_{2}^{*}\right)^{T}$ of the system (24) [31-35].

In addition, we have that condition 3 of Theorem 3 holds, and condition 2 of Theorem 3 is satisfied for

$$
c_{1}=\min _{1 \leq i \leq 2}\left[2\left(\tilde{d}_{i}+\underline{a}_{i} B_{i}\right)-\bar{a}_{i} \sum_{j=1}^{2}\left(L_{j} c_{i j}^{+}+M_{j} w_{i j}^{+}+L_{i} c_{j i}^{+}\right)\right]=4.2
$$

and

$$
c_{2}=\max _{1 \leq i \leq 2}\left(M_{i} \sum_{j=1}^{2} \bar{a}_{j} w_{j i}^{+}\right)=1 .
$$

Therefore, by Theorem 3, we conclude that the set $M=[-\tau, \infty) \times \Omega \times\left\{\mathbb{R}^{2}: u_{i} \leq u_{i}^{*}, i=1,2, \ldots, m\right\}$ is uniformly globally exponentially stable with respect to the impulsive reaction-diffusion CGNN (24). 
Example 2. Consider the uncertain reaction-diffusion CGNN model (28) with uncertain terms as follows:

$$
\left\{\begin{aligned}
\frac{\partial u_{i}(t, x)}{\partial t}= & \sum_{q=1}^{2} \frac{\partial}{\partial x_{q}}\left(D_{i q} \frac{\partial u_{i}(t, x)}{\partial x_{q}}\right) \\
& -\left(a_{i}\left(u_{i}(t, x)\right)+\tilde{a}_{i}\left(u_{i}(t, x)\right)\right)\left[\left(b_{i}\left(u_{i}(t, x)\right)+\tilde{b}_{i}\left(u_{i}(t, x)\right)\right)\right. \\
& -I_{i}(t, x)-\tilde{I}_{i}(t, x) \\
& -\sum_{j=1}^{2}\left(c_{i j}(t)+\tilde{c}_{i j}(t)\right)\left(f_{j}\left(u_{j}(t, x)\right)+\tilde{f}_{j}\left(u_{j}(t, x)\right)\right) \\
& \left.-\sum_{j=1}^{2}\left(w_{i j}(t)+\tilde{w}_{i j}(t)\right)\left(g_{j}\left(u_{j}\left(t-\tau_{j}(t), x\right)\right)+\tilde{g}_{j}\left(u_{j}\left(t-\tau_{j}(t), x\right)\right)\right)\right], t \neq t_{k}, \\
u\left(t_{k}^{+}, x\right)- & u\left(t_{k}, x\right)=\left(\begin{array}{cc}
-2 / 5+\tilde{\gamma}_{1 k} & 0 \\
0 & -1 / 7+\tilde{\gamma}_{2 k}
\end{array}\right) u\left(t_{k}, x\right),
\end{aligned}\right.
$$

where $k=1,2, \ldots$, the continuous functions $\tilde{a}_{i}, \tilde{b}_{i}, \tilde{c}_{i j}, \tilde{w}_{i j}, \tilde{f}_{j}, \tilde{g}_{j}, \tilde{I}_{i}$ and the constants $\tilde{\gamma}_{i k}, i, j=2$ are the uncertain terms.

If all uncertain terms are bounded so that all conditions of Theorem 4 are satisfied and the unknown constants $\tilde{\gamma}_{i k}$ are such that

$$
\tilde{\gamma}_{1 k} \in\left[-\frac{7}{5}, \frac{3}{5}\right], \tilde{\gamma}_{2 k} \in\left[-\frac{8}{7}, \frac{6}{7}\right], k=1,2, \ldots,
$$

then, according to Theorem 4 , the set $M=[-\tau, \infty) \times \Omega \times\left\{\mathbb{R}^{2}: u_{i} \leq u_{i}^{*}, i=1,2, \ldots, m\right\}$ is robustly uniformly globally exponentially stable with respect to the impulsive reaction-diffusion CGNN (25).

\section{Conclusions}

In this paper, the general concept of stability of sets for impulsive reaction-diffusion delayed CGNNs is introduced. Sufficient conditions for uniform global asymptotic stability and uniform global exponential stability with respect to sets are presented. The obtained results are useful in the cases when it is essential to consider attractors other than equilibrium points. A robust stability of sets analysis is also derived for the impulsive reaction-diffusion CGNNs under consideration. Finally, two examples are given to illustrate the effectiveness of the developed approach. The generalized set-stability concept can be extended to study other types of impulsive control neural network delayed systems.

Author Contributions: Conceptualization, G.S. and I.S.; methodology, S.T., G.S., I.S. and C.S.; formal analysis, S.T., G.S., I.S. and C.S.; investigation, S.T., G.S., I.S. and C.S.; writing-original draft preparation, I.S. All authors have read and agreed to the published version of the manuscript.

Funding: This research was funded in part by the European Regional Development Fund through the Operational Program "Science and Education for Smart Growth" under contract UNITe BG05M2OP001-1.001-0004 (2018-2023).

Conflicts of Interest: The authors declare no conflict of interest.

\section{References}

1. Cohen, M.A.; Grossberg, S. Absolute stability of global pattern formation and parallel memory storage by competitive neural networks. IEEE Trans. Syst. Man Cybern. 1983, 13, 815-826. [CrossRef]

2. Guo, S.; Huang, L. Stability analysis of Cohen-Grossberg neural networks. IEEE Trans. Neural Netw. 2006, 17, 106-117. [CrossRef] [PubMed]

3. Meng, Y.; Huang, L.; Guo, Z.; Hu, Q. Stability analysis of Cohen-Grossberg neural networks with discontinuous neuron activations. Appl. Math. Model. 2010, 34, 358-365. [CrossRef] 
4. Wan, A.; Wang, M.; Peng, J.; Mao, W. Global exponential stability analysis of Cohen-Grossberg neural networks. Math. Appl. (Wuhan) 2006, 19, 381-387.

5. Gan, Q. Adaptive synchronization of Cohen-Grossberg neural networks with unknown parameters and mixed time-varying delays. Commun. Nonlinear Sci. Numer. Simul. 2012, 17, 3040-3049. [CrossRef]

6. Song, Q.; Cao, J. Stability analysis of Cohen-Grossberg neural network with both time-varying and continuously distributed delays. Comput. Appl. Math. 2006, 197, 188-203. [CrossRef]

7. Yuan, K.; Cao, J.; Li, H. Robust stability of switched Cohen-Grossberg neural networks with mixed time-varying delays. IEEE Trans. Syst. Man Cybern. 2006, 36, 1356-1363. [CrossRef]

8. Aouiti, C.; Assali, E.A. Nonlinear Lipschitz measure and adaptive control for stability and synchronization in delayed inertial Cohen-Grossberg-type neural networks. Int. J. Adapt. Control 2019. [CrossRef]

9. Ozcan, N. Stability analysis of Cohen-Grossberg neural networks of neutral-type: Multiple delays case. Neural Netw. 2019, 113, 20-27. [CrossRef]

10. Pratap, K.A.; Raja, R.; Cao, J.; Lim, C.P.; Bagdasar, O. Stability and pinning synchronization analysis of fractional order delayed Cohen-Grossberg neural networks with discontinuous activations. Appl. Math. Comput. 2019, 359, 241-260. [CrossRef]

11. Li, R; Cao, J.; Alsaedi, A.; Ahmad, B. Passivity analysis of delayed reaction-diffusion Cohen-Grossberg neural networks via Hardy-Poincarè inequality. J. Franklin Inst. 2017, 354, 3021-3038. [CrossRef]

12. Wang, Z; Zhang, H. Global asymptotic stability of reaction-diffusion Cohen-Grossberg neural networks with continuously distributed delays. IEEE Trans. Neral Netw. 2010, 21, 39-49. [CrossRef] [PubMed]

13. Yan, P.; Lv, T. Periodicity of delayed reaction-diffusion high-order Cohen-Grossberg neural networks with Dirichlet boundary conditions. Rocky Mountain J. Math. 2011, 41, 949-970. [CrossRef]

14. Zhao, H.; Wang, K. Dynamical behaviors of Cohen-Grossberg neural networks with delays and reaction-diffusion terms. Neurocomputing 2006, 70, 536-543. [CrossRef]

15. Chen, W.H.; Liu, L.; Lu, X. Intermittent synchronization of reaction-diffusion neural networks with mixed delays via Razumikhin technique. Nonlinear Dyn. 2017, 87, 535-551. [CrossRef]

16. Lu, J.G. Global exponential stability and periodicity of reaction-diffusion delayed recurrent neural networks with Dirichlet boundary conditions. Chaos Solitons Fractals 2008, 35, 116-125. [CrossRef]

17. Qiu, J. Exponential stability of impulsive neural networks with time-varying delays and reaction-diffusion terms. Neurocomputing 2007, 70, 1102-1108. [CrossRef]

18. Rakkiyappan, R.; Dharani, S.; Zhu, Q. Synchronization of reaction-diffusion neural networks with time-varying delays via stochastic sampled-data controller. Nonlinear Dyn. 2015, 79, 485-500. [CrossRef]

19. Stamova, I.M.; Simeonov, S. Delayed reaction-diffusion cellular neural networks of fractional order: Mittag-Leffler stability and synchronization. J. Comput. Nonlinear Dynam. 2018, 13, 011015-1. [CrossRef]

20. Aouiti, C.; Dridi, F. New results on impulsive Cohen-Grossberg neural networks. Neural Proc. Lett. 2019, 49, 1459-1483. [CrossRef]

21. Bohner, M.; Stamov, G.Tr.; Stamova, I.M. Almost periodic solutions of Cohen-Grossberg neural networks with time-varying delay and variable impulsive perturbations. Commun. Nonlinear Sci. Numer. Simul. 2020, 80, 104952. [CrossRef]

22. Li, X. Exponential stability of Cohen-Grossberg-type BAM neural networks with time-varying delays via impulsive control. Neurocomputing 2009, 73, 525-530. [CrossRef]

23. Li, X. Existence and global exponential stability of periodic solution for impulsive Cohen-Grossberg-type BAM neural networks with continuously distributed delays. Appl. Math. Comput. 2009, 215, 292-307. [CrossRef]

24. Li, L.; Jian, J. Exponential convergence and Lagrange stability for impulsive Cohen-Grossberg neural networks with time-varying delays. J. Comput. Appl. Math. 2015, 277, 23-35. [CrossRef]

25. Benchohra, M.; Henderson, J.; Ntouyas, J. Impulsive Differential Equations and Inclusions, 1st ed.; Hindawi Publishing Corporation: New York, NY, USA, 2006; ISBN: $977594550 X ;$ 978-9775945501.

26. Haddad, W.M.; Chellaboina, V.S.; Nersesov, S.G. Impulsive and Hybrid Dynamical Systems, Stability, Dissipativity, and Control, 1st ed.; Princeton University Press: Princeton, NJ, USA, 2006; ISBN: 9780691127156.

27. Li, X.; Bohner, M.; Wang, C.K. Impulsive differential equations: Periodic solutions and applications. Automatica J. IFAC 2015, 52, 173-178. [CrossRef]

28. Liu, X.; Zhang, K. Impulsive Systems on Hybrid Time Domains, 1st ed.; Springer: Cham, Switzerland, 2019; ISBN: 978-3-030-06211-8; 978-3-030-06212-5. 
29. Stamova, I.M.; Stamov, G.T. Applied Impulsive Mathematical Models, 1st ed.; Springer: Cham, Switzerland, 2016; ISBN: 978-3-319-28060-8; 978-3-319-28061-5.

30. Li, Z.; Li, K. Stability analysis of impulsive Cohen-Grossberg neural networks with distributed delays and reaction-diffusion terms. Appl. Math. Model. 2009, 33, 1337-1348. [CrossRef]

31. Li, K.; Song, Q. Exponential stability of impulsive Cohen-Grossberg neural networks with time-varying delay and reaction-diffusion terms. Neurocomputing 2008, 72, 231-240. [CrossRef]

32. Pan, J.; Liu, X.; Zhong, S. Stability criteria for impulsive reaction-diffusion Cohen-Grossberg neural networks with time-varying delays. Math. Comput. Model. 2010, 51, 1037-1050. [CrossRef]

33. Pan J.; Zhong, S. Dynamical behaviors of impulsive reaction-diffusion Cohen-Grossberg neural network with delays. Neurocomputing 2010, 73, 1344-1351. [CrossRef]

34. Wang, J.L.; Wu, H.N.; Guo, L. Stability analysis of reaction-diffusion Cohen-Grossberg neural networks under impulsive control. Neurocomputing 2013, 106, 21-30. [CrossRef]

35. Zhang, X.; Wu, S.; Li, K. Delay-dependent exponential stability for impulsive Cohen-Grossberg neural networks with time-varying delays and reaction-diffusion terms. Commun. Nonlinear Sci. Numer. Simul. 2011, 16, 1524-1532. [CrossRef]

36. He, W.; Qian, F.; Cao, J. Pinning-controlled synchronization of delayed neural networks with distributed-delay coupling via impulsive control. Neural Netw. 2017, 85, 1-9. [CrossRef] [PubMed]

37. Li, X.; Song, S. Stabilization of delay systems: delay-dependent impulsive control. IEEE Trans. Automat. Control 2017, 62, 406-411. [CrossRef]

38. Li, X.; Wu, J. Sufficient stability conditions of nonlinear differential systems under impulsive control with state-dependent delay. IEEE Trans. Automat. Control 2018, 63, 306-311. [CrossRef]

39. Stamova, I.M. Impulsive control for stability of n-species Lotka-Volterra cooperation models with finite delays. Appl. Math. Lett. 2010, 23, 1003-1007. [CrossRef]

40. Stamova, I.M.; Stamov, A.G. Impulsive control on the asymptotic stability of the solutions of a Solow model with endogenous labor growth. J. Franklin Inst. 2012, 349, 2704-2716. [CrossRef]

41. Stamova, I.; Stamov, G. Mittag-Leffler synchronization of fractional neural networks with time-varying delays and reaction-diffusion terms using impulsive and linear controllers. Neural Netw. 2017, 96, 22-32. [CrossRef]

42. Sun, J.; Qiao, F.; Wu, Q. Impulsive control of a financial model. Phys. Lett. A 2005, 335, 282-288. [CrossRef]

43. Yang, X.; Peng, D.; Lv, X.; Li, X. Recent progress in impulsive control systems. Math. Comput. Simul. 2019, 155, 244-268. [CrossRef]

44. Zhang, X.; Lv, X.; Li, X. Sampled-data based lag synchronization of chaotic delayed neural networks with impulsive control. Nonlinear Dynam. 2017, 90, 2199-2207. [CrossRef]

45. Colace, F.; Loia, V.; Tomasiello, S. Revising recurrent neural networks from a granular perspective. Appl. Soft Comput. 2019, 82, 105535. [CrossRef]

46. Hale, J.K.; Verduyn Lunel, S.M. Introduction to Functional Differential Equations, 1st ed.; Springer: New York, NY, USA, 1993; ISBN: 978-0-387-94076-2, 978-1-4612-8741-4, 978-1-4612-4342-7.

47. Baldwin, S.; Slaminka, E.E. A stable/unstable "manifold" theorem for area preserving homeomorphisms of two dimensions. Proc. Am. Math. Soc. 1990, 109, 823-828.

48. Bernfeld, S.R.; Corduneanu, C.; Ignatyev, A.O. On the stability of invariant sets of functional differential equations. Nonlinear Anal. 2003, 55, 641-656. [CrossRef]

49. Parshad, R.D.; Kouachi, S.; Gutierrez, J.B. Global existence and asymptotic behavior of a model for biological control of invasive species via supermale introduction. Commun. Math. Sci. 2013, 11, 971-992.

50. Li, Z.; Yan, L.; Zhou, X. Global attracting sets and stability of neutral stochastic functional differential equations driven by Rosenblatt process. Front. Math. China 2018, 13, 87-105. [CrossRef]

51. Ruiz del Portal, F.R. Stable sets of planar homeomorphisms with translation preudo-arcs. Discrete Continuous Dynam. Syst. S 2019, 12, 2379-2390. [CrossRef]

52. Skjetne, R.; Fossen, T.I.; Kokotovic, P.V. Adaptive output maneuvering, with experiments, for a model ship in a marine control laboratory. Automatica 2005, 41, 289-298. [CrossRef]

53. Xie, S. Stability of sets of functional differential equations with impulse effect. Appl. Math. Comput. 2011, 218, 592-597. [CrossRef]

54. Liu, B.; Liu, X.; Liao, X. Robust stability of uncertain impulsive dynamical systems. J. Math. Anal. Appl. 2004, 290, 519-533. [CrossRef] 
55. Stamov, G.Tr.; Alzabut, J.O. Almost periodic solutions in the PC-space for uncertain impulsive dynamical systems. Nonlinear Anal. 2011, 74, 4653-4659. [CrossRef]

56. Zecevic, A.I.; Siljak, D.D. Control of Complex Systems: Structural Constraints and Uncertainty, 1st ed.; Springer: New York, NY, USA, 2010; ISBN: 978-1-4419-1215-2, 978-1-4614-2555-7, 978-1-4419-1216-9.

57. Zhang, X.; Li, X.; Cao, J.; Miaadi, F. Design of memory controllers for finite-time stabilization of delayed neural networks with uncertainty. J. Franklin Inst. 2018, 355, 5394-5413. [CrossRef]

58. Vidhya, C.; Balasubramaniam, P. Robust stability of uncertain Markovian jumping stochastic Cohen-Grossberg type BAM neural networks with time-varying delays and reaction diffusion terms. Neural Parallel Sci. Comput. 2011, 19, 181-196.

(C) 2019 by the authors. Licensee MDPI, Basel, Switzerland. This article is an open access article distributed under the terms and conditions of the Creative Commons Attribution (CC BY) license (http://creativecommons.org/licenses/by/4.0/). 